Internist 2014 · 55:342-342

DOI 10.1007/s00108-014-3458-1

Online publiziert: 22. Februar 2014

(c) Springer-Verlag Berlin Heidelberg 2014
J. Dinger ${ }^{1} \cdot$ S. Nitschmann ${ }^{2}$

${ }^{1}$ Zentrum für Epidemiologie und Gesundheitsforschung Berlin

${ }^{2}$ Werl-Westönnen

\title{
Erratum zu: Kardiovaskuläre Auswirkungen hormoneller Kontrazeption
}

In der gedruckten sowie der HTML-Version des Beitrags wurden bedauerlicherweise Textpassagen nicht abgedruckt. Der vollständige Text des entsprechenden Abschnitts lautet:

\section{Ergebnisse}

Es wurden 1.626.158 Frauen in die Studie eingeschlossen. Insgesamt ereigneten sich während der 14.251.063 Personenjahre 3311 thrombotische Schlaganfälle (1633 zerebrale Infarkte und 1678 zerebrale Apoplexe) und 1725 Herzinfarkte. Es verstarben 34 der 3311 Schlaganfallpatientinnen und 186 der 1725 Myokardinfarktpatientinnen (1,0\% bzw. 10,8\%).

Die Endpunkthäufigkeitsraten in Abhängigkeit vom Ethinylestradiol- und Progesterongehalt p.o. sind in Tab. 1 dargestellt. Keines der alleinigen Progesteronprodukte, einschließlich der intrauterinen bzw. subkutanen Applikation, erhöhte das thromboembolische Risiko, während das Risiko bei der Verwendung von kontrazeptiven Patches und Vaginalringen deutlich erhöht war (relatives Risiko: 3,15 bzw. 2,49).

Die Inzidenz thromboembolischer Ereignisse stieg mit zunehmendem Alter um das 20-Fache (15- bis 19-Jährige vs. 45 - bis 49-Jährige) und sank mit zunehmendem Bildungsstand. Deutlich erhöht war das Risiko bei Patienten mit Diabetes mellitus, Hypertonie, Hyperlipidämie, Arrhythmie und Nikotinabusus. Informationen zum Raucherstatus lagen von 480.223 Frauen vor (5,2 Mio. Personenjahre). 582 Frauen mit Schlaganfall und 193 mit Myokardin- farkt waren Raucherinnen (relatives Risiko: 1,57 bzw. 3,62).

\section{Kommentar \\ Dr. J. Dinger \\ Zentrum für Epidemiologie und Gesundheitsfor- schung Berlin}

\section{Bisheriger Erkenntnisstand}

Kombinationen von Östrogenen und Gestagenen erhöhen das Risiko von venösen Thromboembolien. Dies gilt unabhängig davon, ob diese Kombinationen zur Empfängnisverhütung oder zur Hormontherapie eingesetzt werden. Die Studienlage bei arteriellen Thromboembolien ist weniger eindeutig. Während die Mehrzahl der Studien auf ein erhöhtes Risiko thrombotischer Schlaganfälle hinweist, gibt es nur einzelne Studien, die verwertbare Hinweise auf ein erhöhtes Herzinfarktrisiko liefern. Insgesamt legt die bisherige Studienlage nahe, dass hormonelle Kombinationspräparate zur Empfängnisverhütung das kardiovaskuläre Mortalitätsrisiko aufgrund von arteriellen Thromboembolien erhöhen [4]. Allerdings ist das den Kontrazeptiva zurechenbare absolute Risiko bei Nichtraucherinnen unter 35 Jahren so gering, dass in den meisten Fällen keine Bedenken gegen eine Verschreibung bestehen. Deutlich höher ist das zurechenbare Risiko bei Raucherinnen ab 35 Jahren. Dementsprechend gilt in den USA und Japan diese Konstellation als Kontraindikation. Auch wenn es sich hierbei nach wissenschaftlichen Kriterien nicht um zweifelsfrei gesichertes Wissen handelt, stellt diese Interpretation der Daten- lage - im Sinne des Patientenschutzes auch in Europa die Richtschnur für ärztliche Nutzen-Risiko-Abwägungen dar.

Der Verlag bittet, den Fehler zu entschuldigen.

Die Redaktion

\section{Korrespondenzadresse}

Dr. J. Dinger

Zentrum für Epidemiologie und Gesundheitsforschung Berlin Invalidenstr. 115, 10115 Berlin dinger@zeg-berlin.de 